\title{
Die Deutsche CML-Allianz: Ein Modell für intersektorale Kommunikation und Kooperation
}

Jährlich erkranken rund 1200 Menschen in Deutschland an der chronischen myeloischen Leukämie (CML). Die Einführung von Tyrosinkinase-Inhibitoren (TKI) hat die Prognose dieser Patienten deutlich verbessert. Bei einer Gesamtsterblichkeit von etwa $1,6 \%$ pro Jahr unter Imatinib-Therapie steigt die Prävalenz der Erkrankung stetig an. Mit den Inhibitoren der zweiten Generation, Nilotinib und Dasatinib, werden raschere und tiefere molekulare Remissionen erzielt. Mit Bosutinib und Ponatinib stehen weitere Inhibitoren für klinische Problemsituationen zur Verfügung. Bei unterschiedlicher Spezifität für BCR-ABL und andere Tyrosinkinasen unterscheidet sich auch das Nebenwirkungsprofil der einzelnen TKI [1]. Unverzichtbarer Bestandteil des Managements von CML-Patienten ist die leitliniengerechte Anwendung der Zytogenetik, der standardisierten quantitativen PCR und der Mutationsdiagnostik zur Beurteilung des Remissionsstatus und der frühzeitigen Aufklärung einer drohenden Resistenz [2].

Die Deutsche CML-Studiengruppe hat in den letzten 32 Jahren systematisch große Therapieoptimierungsstudien durchgeführt, in die bisher mehr als 4500 Patienten aufgenommen werden konnten [3]. Sorgfältige Analysen zeigten, dass die Therapie innerhalb einer klinischen Studie das Gesamtüberleben der Patienten verbessert und insbesondere der rechtzeitige Zugang zu Zweitlinientherapien einen entscheidenden Parameter für eine gute Prognose darstellt [4].

Langjährige therapiefreie Remissionen bei Patienten in klinischen Studien lassen die Hoffnung auf eine Heilbarkeit der CML aufkommen. Parallel zur oder im Anschluss an die TKI-Therapie appliziertes Interferon- $\alpha$ induziert eine Immunantwort gegen den leukämischen Klon [5]. Mit dem Ziel der Verbesserung und der Stabilisie- rung der Remission nach dem Absetzen der Therapie wird dies gegenwärtig im Rahmen der TIGER-Studie prospektiv untersucht. Um allen CML-Patienten in Deutschland ein optimales Management ihrer Erkrankung zu ermöglichen, wurde im Oktober 2014 die Deutsche CML-Allianz gegründet. Ziel dieser Initiative der CML-Studiengruppe und des Arbeitskreises klinische Studien e.V. ist es, eine flächendeckende Versorgung der Patienten nach modernen Standards zu gewährleisten. Die koordinierte Zusammenarbeit aller über 300 mit der CML-Behandlung betrauten Institutionen, also der Universitätskliniken, Krankenhäuser, onkologischen Schwerpunktpraxen und Labore, sowie die Einbeziehung der Patientenvertreter ermöglichen den frühen Einsatz neuer Therapieoptionen bei Risikopatienten.

Beim Management der Erkrankung sind eine optimale Verlaufskontrolle [6, 7], die Erkennung von Problemsituationen sowie eine frühzeitige Therapieoptimierung im Bedarfsfall besonders wichtig. Dies geschieht bislang häufig nur in den größeren Zentren im Rahmen von klinischen Studien. Mit der CML-Allianz sollen die Möglichkeiten weiter ausgebaut werden und allen Patienten, unabhängig vom Wohn- oder Behandlungsort, der Zugang zur bestmöglichen Information, zur standardisierten Diagnostik und zu neuen Therapien ermöglicht werden.

Die Deutsche CML-Allianz ist in 4 Bereichen aktiv. Dazu zählen i) der breitere $\mathrm{Zu}$ gang zu neuen Therapieansätzen im Rahmen klinischer Studien, darauf aufbauend ii) eine gemeinsame Qualitätssicherung, iii) die Zusammenarbeit und der Austausch bei der standardisierten Labordiagnostik und iv) gemeinsame Informations- und Weiterbildungsprogramme für Ärzte, Pflegekräfte, Studienassistenten und Patienten.

Die Arbeit der CML-Allianz wird von einer Leitgruppe unter Vorsitz von Prof. Dr. Andreas Hochhaus, Jena, und Prof. Dr.
Hans Tesch, Frankfurt, koordiniert. Vier Tutoren sorgen für den niederschwelligen Zugang zu klinischen Studien für Kollegen. Jeweils im Frühjahr findet ein Jahrestreffen der CML-Allianz in Weimar statt. Weiterhin gibt es dezentrale Veranstaltungen der CML-Allianz in allen Regionen Deutschlands und Treffen während den Jahrestagungen der Deutschen, Österreichischen und Schweizerischen Gesellschaften für Hämatologie und Onkologie 2015 in Basel und 2016 in Leipzig.

Die Allianz steht allen am Management der CML Beteiligten zur Mitarbeit offen. Im Blickpunkt steht eine «therapiefreie Remission» für die Mehrheit der Patienten.

Diese erste Ausgabe in 2015 rückt chronische Leukämien in den Mittelpunkt. Doch nicht nur deswegen ist die CML-Allianz an dieser Stelle zu erwähnen, sondern auch aufgrund ihres Ansatzes, die Behandler in allen Bereichen zu vernetzen, wodurch Praktiker und Kliniker einbezogen werden. Somit ist das Projekt verwandt mit dem Wissenstransfer-Konzept von Karger Kompass OnKologie und stößt bei Ihnen - liebe Leser - hoffentlich auf besonderes Interesse.

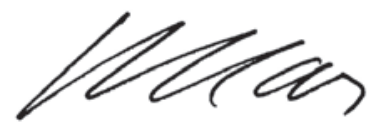

Prof. Dr. Andreas Hochhaus

\section{Referenzen}

1 Hochhaus A, et al: Internist (Berl) 2015;56: 333-343.

2 Baccarani M, et al: Blood 2013;122:872-884.

3 Kalmanti L, et al: Leukemia 2015: doi: 10.1038/ leu.2015.36.

4 Lauseker M, et al: Blood 2014;123:2494-2496.

5 Burchert A, et al: Leukemia 2015: doi: 10.1038/ leu.2015.45.

6 Hanfstein B, et al: Leukemia 2012;26: 2096-2102.

7 Cross NC, et al: Leukemia 2015: doi: 10.1038/ leu.2015.29.

\section{KARGER}

Fax +4976145207 14

information@karger.com

www.karger.com (c) 2015 S. Karger GmbH, Freiburg 2296-5416/15/0021-0006\$39.50/0

Accessible online at: www.karger.com/kko
Prof. Dr. Andreas Hochhaus

Klinik für Innere Medizin II, Abteilung Hämatologie und Internistische Onkologie Universitätsklinikum Jena

Erlanger Allee 101, 07747 Jena, Deutschland

andreas.hochhaus@med.uni-jena.de 